\title{
Association of dynamic changes in serum cytokine levels with the severity of injury in patients suffering from closed chest traumas complicated with pulmonary contusions
}

\author{
KEQIANG LIU, JIFU LIU and SHANSHAN WU \\ Department of Thoracic Surgery, PLA General Hospital of Beijing Military Area, Beijing 100700, P.R. China
}

Received January 13, 2011; Accepted March 16, 2011

DOI: $10.3892 /$ etm.2011.241

\begin{abstract}
This study aimed to examine the dynamic changes in the serum levels of tumor necrosis factor (TNF)- $\alpha$, interleukin (IL)-6 and IL-10 in patients suffering from closed chest injuries and concomitant pulmonary contusions and their correlation with the Thoracic Trauma Severity Score (TTS) and Injury Severity Score (ISS). Patients with multiple traumas with an ISS $\geq 16$ ( $n=41)$ were evaluated in the study. Patients included 21 individuals with trauma complicated by pulmonary contusion and 20 patients with trauma without contusion. Venous blood samples were collected from the patients on days $1,2,3,5$ and 7 following trauma to assess serum levels of TNF- $\alpha$, IL- 6 and IL-10, whose correlation with TTS and ISS was analyzed. Levels of TNF- $\alpha$, IL-6 and IL-10 remained significantly higher in the patients with trauma complicated by contusion compared to the patients with trauma without contusion $(\mathrm{P}<0.01)$. These three cytokines were positively correlated with TTS and ISS in the patients with trauma complicated by contusion. Serum TNF- $\alpha$, IL-6 and IL-10 levels increased significantly in the patients with trauma complicated by pulmonary contusion. In conclusion, both cytokine assays and TTS facilitate the accurate assessment of the severity of chest injury.
\end{abstract}

\section{Introduction}

Pulmonary contusion occurs when an external force is exerted on lung tissues, which is usually associated with a closed chest injury frequently resulting from a traffic accident, free fall from a height or blunt trauma. The strong force on the chest wall reduces the thoracic volume and consequently increases intrathoracic pressure, which compresses lung tissues, leading to pulmonary parenchymal hemorrhage and edema, pulmo-

Correspondence to: Dr Keqiang Liu, Department of Thoracic Surgery, PLA General Hospital of Beijing Military Area, Beijing 100700, P.R. China

E-mail: ylkq0402@sina.com

Key words: tumor necrosis factor- $\alpha$, interleukin-6, interleukin-10, multiple trauma, pulmonary trauma nary capillary injuries, interstitial and alveolar effusion, interstitial edema and the infiltration of inflammatory cells. These pathological changes result in decreased ventilation and gas exchange, severe ventilation-perfusion imbalance, hypoxia and poor pulmonary compliance. Pulmonary contusion is one of the major causes of acute lung injury and acute respiratory distress syndrome and can develops into systemic inflammatory response syndrome and multiple organ dysfunction syndrome, resulting in a high incidence of mortality. Recently, numerous theoretical and clinical studies on pulmonary contusion have been carried out, particularly concerning the role of inflammatory cytokines. Following contusion, lung tissues are exposed to a potent inflammatory response in which alveolar epithelial and endothelial cells become extensively degenerated and necrotic, compromising the gas exchange in alveoli. This inflammatory pathogenesis can be accelerated by inflammatory cytokines into a response cascade. Moreover, cytokines are believed to play their roles by interacting through a complex network.

The evaluation of trauma severity is critical for the diagnosis, treatment and prediction of prognosis of the trauma patient. Based on the Abbreviated Injury Scale (AIS), the Injury Severity Score (ISS) is the most commonly used hospital trauma scoring system and plays an important role in trauma rescue. ISS includes the comprehensive evaluation of multiple traumas by a general overview of anatomical injury and predicts the probability of patient survival. However, this scoring system has its innate limits in clinical practice. For example, for a single site of multiple trauma, the maximum AIS (mAIS) alone does not reflect the severity of the injury. In addition to ISS, a specific chest injury scoring system is required for assessment of patients with multiple trauma complicated by chest injury. Pape et al (4) proposed the Thoracic Trauma Severity Score (TTS) for the early evaluation of chest injury. The primary measures of TTS consist of the severity of chest and thoracic injury, oxygenation index and patient age. The use of TTS, as a newly emerging chest injury scoring system in clinical practice to supplement other currently available scoring tools, should be justified in further clinical studies.

In the present study, we examined the dynamic changes in the serum levels of tumor necrosis factor (TNF)- $\alpha$ interleukin (IL)-6 and IL-10 in patients suffering from closed chest injuries and concomitant pulmonary contusions. Additionally, changes in pro- and anti-inflammatory cytokines were assessed. 
Table I. General characteristics of the patients with or without pulmonary contusion.

With contusion $(n=21)$

Without contusion $(n=20)$

\begin{tabular}{lcc}
\hline Gender (M/F) & $16 / 5$ & $17 / 3$ \\
Age, mean (range), in years & $45.62 \pm 17.74(21-85)$ & $48.30 \pm 12.04(20-73)$ \\
Inclusion criteria & i) ISS $\geq 16$; ii) Shows patchy and obscure shadows on & i) ISS $\geq 16$ \\
chest radiograph and CT scan within 24 h of trauma & $29.95 \pm 7.89(18-48)$ \\
ISS (range) & $29.00 \pm 8.26(17-50)$ & $3.57 \pm 1.99(1-7)$ \\
TTS (range) & $9.76 \pm 2.79(5-16)$ & 17 \\
Cause of trauma & 13 & 2 \\
Traffic accident & 4 & 1 \\
Free fall from injury & 4 & \\
Heavy weight injury & & \\
\hline
\end{tabular}

Table II. Changes in the serum TNF- $\alpha$, IL-6 and IL-10 levels (mean \pm SD) in the trauma patients.

\begin{tabular}{llccc}
\hline $\begin{array}{l}\text { Post-trauma } \\
\text { time (days) }\end{array}$ & \multicolumn{1}{c}{ Group } & TNF- $\alpha(\mathrm{pg} / \mathrm{ml})$ & $\mathrm{IL}-6(\mathrm{pg} / \mathrm{ml})$ & $\mathrm{IL}-10(\mathrm{pg} / \mathrm{ml})$ \\
\hline & & & \\
1 & Control & $25.46 \pm 5.08$ & $17.55 \pm 0.97$ & $10.37 \pm 5.12$ \\
1 & With contusion & $130.16 \pm 38.12^{\mathrm{a}, \mathrm{b}}$ & $102.28 \pm 31.50^{\mathrm{a}, \mathrm{b}}$ & $95.06 \pm 29.86^{\mathrm{a}, \mathrm{b}}$ \\
2 & Without contusion & $97.63 \pm 21.12$ & $77.35 \pm 18.39$ & $71.25 \pm 19.19$ \\
2 & With contusion & $113.77 \pm 33.85^{\mathrm{a}, \mathrm{b}}$ & $117.18 \pm 34.18^{\mathrm{a}, \mathrm{b}}$ & $108.86 \pm 30.98^{\mathrm{a}, \mathrm{b}}$ \\
3 & Without contusion & $82.18 \pm 19.84$ & $89.78 \pm 19.74$ & $83.62 \pm 21.23$ \\
3 & With contusion & $98.26 \pm 38.95^{\mathrm{a}, \mathrm{b}}$ & $89.44 \pm 25.25^{\mathrm{a}, \mathrm{b}}$ & $84.12 \pm 24.00^{\mathrm{a}, \mathrm{b}}$ \\
5 & Without contusion & $67.92 \pm 17.54$ & $61.10 \pm 16.96$ & $54.13 \pm 15.39$ \\
5 & With contusion & $76.00 \pm 24.18^{\mathrm{a}, \mathrm{b}}$ & $73.53 \pm 32.49^{\mathrm{a}, \mathrm{b}}$ & $66.59 \pm 18.95^{\mathrm{a}, \mathrm{b}}$ \\
7 & Without contusion & $50.84 \pm 13.84$ & $45.72 \pm 11.92$ & $39.66 \pm 9.96$ \\
7 & With contusion & $55.95 \pm 16.94^{\mathrm{a}, \mathrm{b}}$ & $48.39 \pm 14.40^{\mathrm{a}, \mathrm{b}}$ & $40.31 \pm 10.84^{\mathrm{a}, \mathrm{b}}$ \\
\hline
\end{tabular}

Patients with pulmonary contusion vs. healthy control patients, ${ }^{\mathrm{a}}<0.01$; vs. patients without pulmonary contusion, ${ }^{\mathrm{b}} \mathrm{P}<0.01$.

Differences in cytokine levels between patients with and without pulmonary contusion sharing similar ISS were determined, and the correlation of cytokine levels with trauma scores was analyzed. The assay of cytokine levels in combination with TTS and ISS may be an effective tool for evaluating the severity of injury in patients suffering from closed chest injury and concomitant pulmonary contusions. Justification of the use of TTS in clinical practice for the estimation of chest injury as a supplementary measure to the systemic trauma evaluation system was herein attempted.

\section{Patients and methods}

Patient characteristics. Patients suffering from multiple trauma $(n=41)$ who were hospitalized in our surgical unit between January 2007 and January 2008 were studied. The patients included 33 males and 8 females, aged 20-85 years (mean 46.93 \pm 15.10 ). The cause of trauma consisted of traffic accident $(n=30)$, free fall from a height $(n=6)$ and heavy weight blunt injury $(n=5)$. The inclusion criterion of multiple trauma patients was ISS $\geq 16$. Twenty-one patients were afflicted with closed chest injury and pulmonary contusion, whereas the remaining 20 patients were not complicated by pulmonary contusion. The two groups did not differ significantly in ISS. The general characteristics of the two groups are shown in Table I. No deaths occurred during hospitalization. Healthy volunteers $(n=15)$ were enrolled simultaneously as controls, including 12 males and 3 females (mean age 47.67 \pm 17.06 years; range 21-80).

Sample collection and measurement. Venous blood samples $(5 \mathrm{ml})$ were collected from all of the post-traumatic patients on days 1,2,3, 5 and 7 following trauma, whereas peripheral venous blood samples were also collected from fasting control patients simultaneously. Blood samples were separated at room temperature and stored at $-20^{\circ} \mathrm{C}$ for further batch assay using an avidin-biotin complex ELISA kit as recommended by the manufacturer.

Statistical analysis. Data were processed by using SPSS 13.0 statistical analysis software. The quantitative data were expressed as the means \pm SD for the analysis of variance and correlation analysis. $\mathrm{P}<0.05$ was considered statistically significant, whereas $\mathrm{P}<0.01$ was highly significant. 


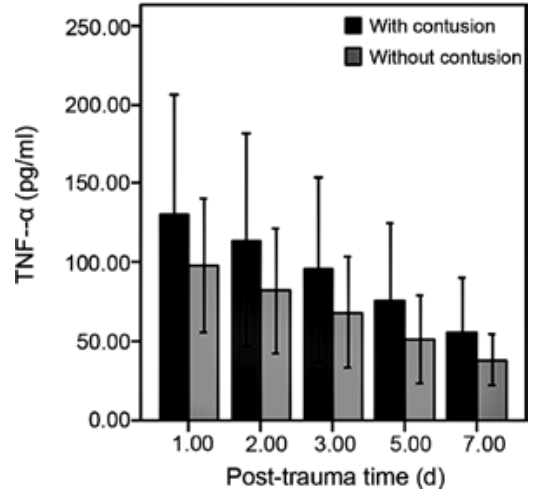

Figure 1. Changes in serum TNF- $\alpha$ levels in patients with or without contusion.

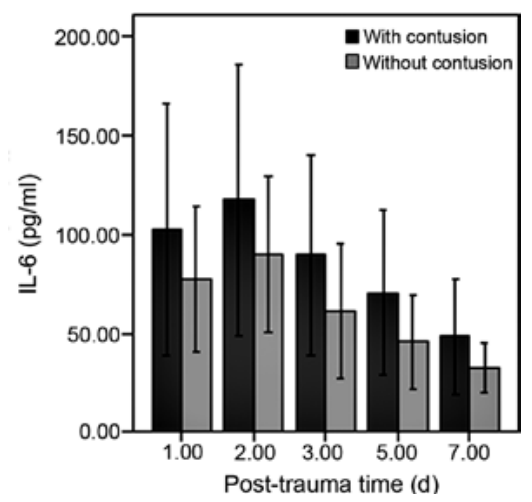

Figure 2. Changes in serum IL-6 levels in patients with or without contusion.

\section{Results}

Statistical analyses showed that the serum levels of TNF- $\alpha$, IL-6 and IL-10 in patients with traumas complicated by pulmonary contusion were significantly higher than those in patients without pulmonary contusion and healthy control patients on days $1,2,3,5$ and 7 after trauma (both $\mathrm{P}<0.01$ ) (Table II).

The serum TNF- $\alpha$ level significantly increased and reached a peak in the patients with traumas complicated by pulmonary contusion within 1 day after trauma, and these levels exhibited a progressively decreasing trend throughout days 2, 3, 5 and 7 (Fig. 1). The serum levels of IL-6 and IL-10 were significantly increased within 1 day after trauma, both of which generally reached peaks on day 2, whereas both markers decreased daily throughout days 3,5 and 7 after trauma (Figs. 2 and 3). On day 7 after trauma, the levels of the three cytokines were still higher than the normal limits (Fig. 4).

The correlation analysis showed that in patients with traumas complicated by pulmonary contusion, the serum levels of TNF- $\alpha$ (Figs. 5 and 6), IL-6 (Figs. 7 and 8) and IL-10 (Figs. 9 and 10) were positively correlated with TTS and ISS in a statistically significant manner.

\section{Discussion}

Patients presenting with closed chest injuries are usually in a serious condition when their injury is complicated by pulmonary contusion and consequently are associated with a high incidence of mortality. A potent inflammatory response

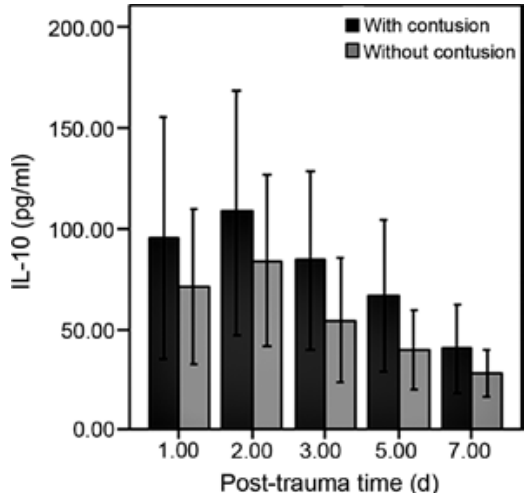

Figure 3. Changes in serum IL-10 levels in patients with or without contusion.

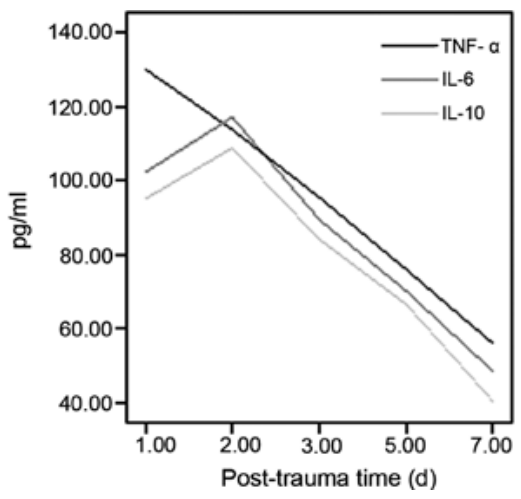

Figure 4. Dynamic changes in serum TNF- $\alpha$, IL-6 and IL-10 levels in patients with contusion.

occurs in contused lung tissues, where inflammatory cytokines aggravate pre-existing injuries through a cascade of reactions. Cytokines are known to interact with each other, forming a complex cytokine network, which plays an important role in the response to post-traumatic inflammatory and immune imbalance (1).

TNF- $\alpha$ is a crucial inflammatory cytokine involved in the response to severe trauma and demonstrates extensive biological activity. TNF- $\alpha$ induces the release of other cytokines, such as IL-6, which mediates the post-traumatic inflammatory response and exacerbates the injuries to tissues and organs. IL- 6 is synthesized in response to the stimulation of TNF- $\alpha$, and has no harmful effect on cells itself, yet promotes the activation and aggregation of neutrophils, reflecting the severity of tissue injury. IL-10 is a superpotent, immunocompromising and anti-inflammatory cytokine which plays an important role in the regulation of the cytokine network. IL-10 inhibits the release of TNF- $\alpha$, IL-1, IL- 6 and INF- $\gamma$ from monocytes, which are stimulated to produce an IL-1 receptor inhibitor in an anti-inflammatory manner. The expression of IL-10 demonstrates a linear correlation with the expression levels of TNF- $\alpha$, IL- 6 and IL-8. The progression of injury and inflammation increases the responsiveness of TNF- $\alpha$, IL- 6 and IL-8, whereas anti-inflammatory cytokines, IL-1ra, IL-4 and IL-10, increase simultaneously to antagonize the high levels of pro-inflammatory factors. The rise in serum IL-10 in post-traumatic patients may compromise immunity and impair resistance to infection (2). The present study 


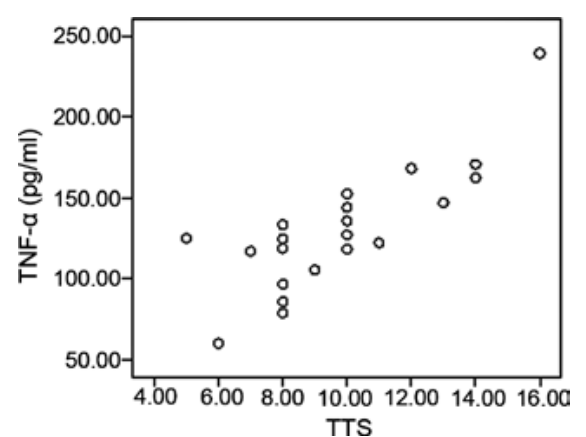

Figure 5. Correlation analysis between TNF- $\alpha$ peak values and TTS scores $(\mathrm{r}=0.844)$.

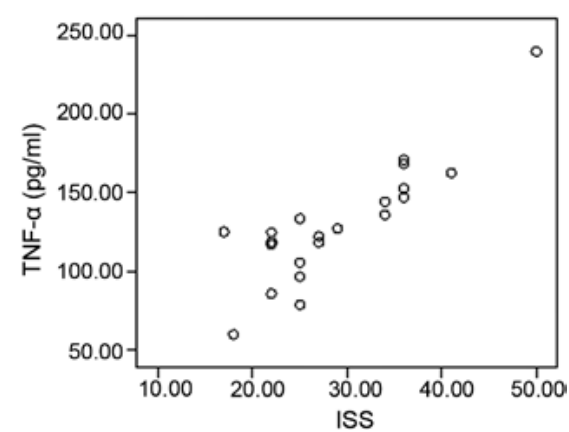

Figure 6. Correlation analysis between TNF- $\alpha$ peak values and ISS scores $(\mathrm{r}=0.812)$.

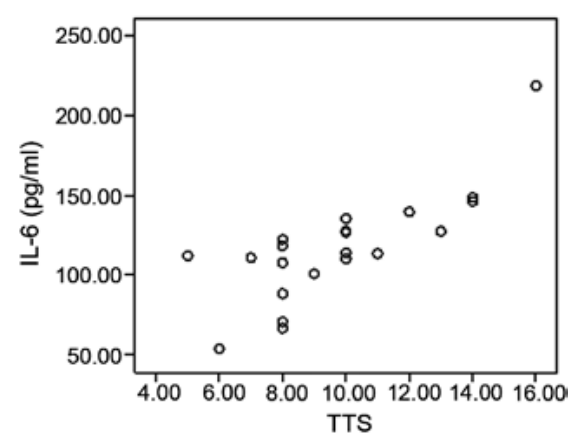

Figure 7. Correlation analysis between IL-6 peak values and TTS scores $(\mathrm{r}=0.776)$.

demonstrated that serum IL-10 reached a peak within 2 days after trauma, suggesting the onset of an immune imbalance.

In the present study, TNF- $\alpha$ was found to be released immediately following trauma. The level of TNF- $\alpha$ peaked on day 1 after trauma and initiated an inflammatory response. IL- 6 and IL-10 were released at a later time point and their levels peaked on day 2 , but decreased progressively afterwards. Levels of the above three cytokines remained significantly higher 1 week after trauma $(\mathrm{P}<0.01)$. Perl et al (3) examined the levels of IL-6, IL-8, IL-10 and IL-12 in lungs, skeleton, skin, subcutaneous fat and muscle in patients suffering from a single trauma and found the highest level in lung tissues. Our study also detected a significant difference in serum TNF- $\alpha$, IL- 6 and IL-10 levels among trauma patients with pulmonary contusion, trauma patients without contusion and healthy volunteers $(\mathrm{P}<0.01)$. However, those with and

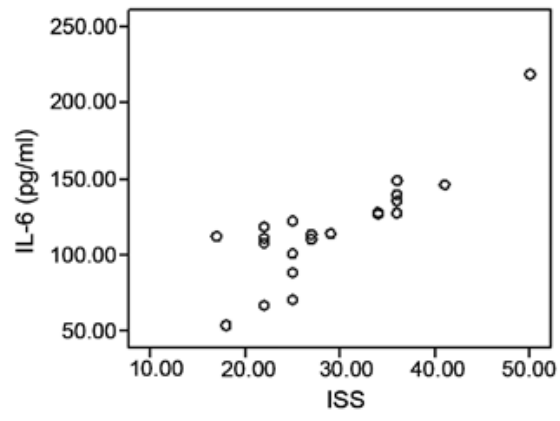

Figure 8 . Correlation analysis between IL-6 peak values and ISS scores $(\mathrm{r}=0.824)$.

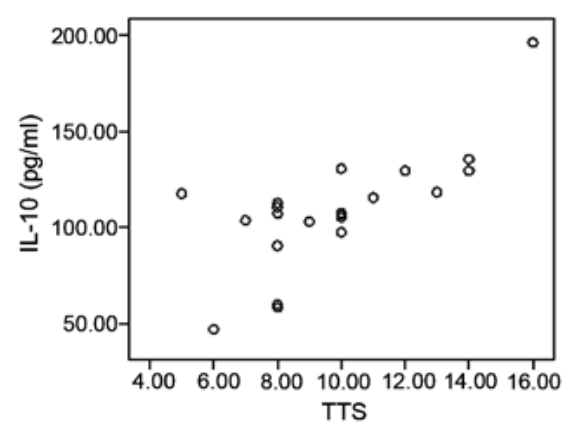

Figure 9. Correlation analysis between IL-10 peak values and TTS scores $(\mathrm{r}=0.661)$.

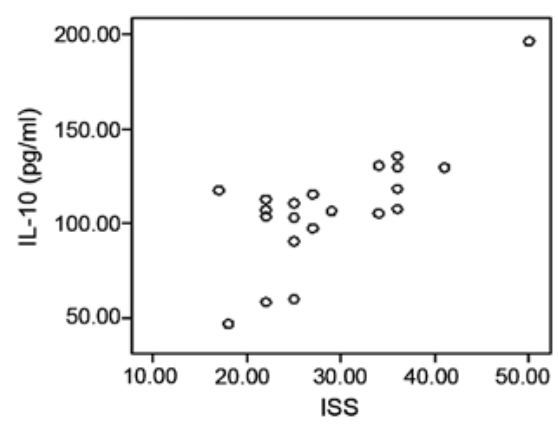

Figure 10. Correlation analysis between IL-10 peak values and ISS scores $(\mathrm{r}=0.627)$.

without contusion did not differ significantly in regards to ISS, suggesting that under comparable systematic stress, patients with injuries complicated by pulmonary contusion had a more serious inflammatory response and higher inflammatory cytokine levels.

ISS is the most commonly used hospital evaluation tool of trauma, and a higher score indicates a more severe injury and a higher risk of mortality. It is common clinical practice to regard ISS $\geq 16$ as severe injury. All of the studied multiple trauma patients had an ISS $\geq 16$. Due to its limitations, the ISS does not reflect pathological changes in patients and the effects of health and age on injury, nor does it discriminate the severity of injury in patients with comparable ISS or the difference between a single injury and multiple injuries in a specific site. Inclusion of the three most seriously injured areas separately is likely to underestimate the severity of multiple 
injuries in a specific site. In the present study, 5 patients suffered from bilateral pulmonary contusions, whereas ISS only considered the unilateral condition, failing to accurately reflect the injury. In patients concomitantly complicated by multiple traumas and pulmonary contusion, ISS cannot accurately assess the actual severity of injury. A specific scoring system targeting chest injury is thus required. Pape et al (4) established an early scoring system for chest injury, namely TTS, through the analysis of 1,495 multiple trauma patients, which was rarely reported in patients with injuries complicated with pulmonary contusion. The major measures of TTS include the severity of chest and thoracic injury, oxygenation index, patient age and bilateral injury. Such a scoring system indicates that pulmonary contusion has a more significant effect on prognosis than rib fracture. However, in patients over 70 years of age, rib fracture is a worse prognostic factor than pulmonary contusion, and those with bilateral injuries have an even higher risk of mortality. The severity of pulmonary contusion is closely associated with prognosis; thus, development of an objective evaluation tool is necessary. The severity of pulmonary contusion should be evaluated within $24 \mathrm{~h}$, based on imaging data and oxygenation index.

Tyburski et al (5) established a pulmonary contusion scoring system based on chest radiographs, where one third of the unilateral pulmonary field was appraised as 3 points and entire involvement of the unilateral field as 9 points. In this system, a score of less than 3 points was evaluated as mild chest injury, 3-9 points as moderate and 10-18 points as severe chest injury. Chest CT scan has a higher sensitivity and specificity than chest radiography, and is a better estimate of the percent volume of contused lung tissue in relation to the entire lung. It is also more effective for identifying fractures of the sternum, thoracic vertebrae and scapulae which are easily missed on radiographs (6). Offner and Moore (7) characterized the severity of pulmonary contusion according to grade $0-3$ based on the ratio of $\mathrm{PaO}_{2} / \mathrm{FiO}_{2}$, where grade 0 referred to a ratio $>250$, grade $1,175-250$, grade 2, 100-174 and grade 3, $<100$. Further clinical analysis confirmed that such a ratio was an important measure for predicting mortality secondary to pulmonary contusion.

In a simple pulmonary contusion animal model, the most massive pathological response was found to occur within $8 \mathrm{~min}$ to $24 \mathrm{~h}$ following trauma, when arterial oxygenation decreased aggressively and the IL-6 level in bronchial alveolar lavage fluid increased rapidly to a peak level (8). Kang et al (9) reported that in traumatized rodents, middleaged animals exhibited higher TNF- $\alpha$, IL- 6 and IL-10 levels than young animals, suggesting the association of cytokine secretion with age. Wichmann et al (10) reported that patients with hemorrhagic bone fractures and cartilage injuries secreted more inflammatory cytokines than those complicated without hemorrhage, showing an immunocompromised immunity, suggesting the effect of hemorrhage on the secretion of inflammatory cytokines. TSS involves measures of the traumatized anatomical site in addition to patient age, posttrauma physiological index and signs in a comprehensive and objective manner.

In patients dying from serious injury, serum pro-inflammatory cytokines, such as IL-6 and IL-8, remained at a higher level than those in surviving patients in a relationship proportional to the severity of the injury (11). Taniguchi et al (12) reported that the ratio of IL-6/IL-10 was positively correlated with ISS in patients suffering from thoracic and abdominal injuries. This ratio was found to be a prognostic factor; a higher ratio was associated with poor prognosis and a lower ratio with a more favorable prognosis. The present study demonstrated that pro-inflammatory cytokines, such as TNF- $\alpha$ and IL-6, and anti-inflammatory cytokines, such as IL-10, were positively correlated with both ISS and TTS. Similar to ISS, TTS better reflected the severity of injury in a comprehensive and objective manner, specifically evaluating the severity of chest injury, independent of the conditions of other sites.

In the present study, serum inflammatory cytokines increased significantly in patients concomitantly suffering from closed chest injury and pulmonary contusion, showing a stronger inflammatory response. TSS may be used as an evaluation tool to accurately assess the severity of chest injury. For patients with multiple trauma dominantly involving chest injury, the evaluation of injury severity should incorporate ISS and TTS, which can characterize the systemic injury and local condition. The dynamic monitoring of plasma TNF- $\alpha$, IL-6 and IL-10 has a significant role in the prediction of the clinical outcome of patients with closed chest injuries with pulmonary contusions.

\section{References}

1. Dinarello CA: Proinflammatory and anti-inflammatory cytokines as mediators in the pathogenesis of septic shock. Chest 112: S321-S329, 1997.

2. Lyons A, Goebel A, Mannick JA and Lederer JA: Protective effects of early interleukin 10 antagonism on injury-induced immune dysfunction. Arch Surg 134: 1317-1324, 1999.

3. Perl M, Gebhard F, Knoferl MW, Bachem M, Gross HJ, Kinzl L and Strecker W: The pattern of preformed cytokines in tissues frequently affected by blunt trauma. Shock 19: 299-304, 2003.

4. Pape HC, Remmers D, Rice J, Ebisch M, Krettek C and Tscherne H: Appraisal of early evaluation of blunt chest trauma: development of a standardized scoring system for initial clinical decision making. J Trauma 49: 496-504, 2000.

5. Tyburski JG, Collinge JD, Wilson RF and Eachempati SR: Pulmonary contusions: quantifying the lesions on chest X-ray films and the factors affecting prognosis. J Trauma 46: 833-838, 1999.

6. Traub M, Stevenson M, McEvoy S, Briggs G, Lo SK, Leibman S and Joseph T: The use of chest computed tomography versus chest X-ray in patients with major blunt trauma. Injury 38: 43-47, 2007.

7. Offner PJ and Moore EE: Lung injury severity scoring in the era of lung protective mechanical ventilation: the $\mathrm{PaO}_{2} / \mathrm{FIO}_{2}$ ratio. J Trauma 55: 285-289, 2003.

8. Raghavendran K, Davidson BA, Woytash JA, et al: The evolution of isolated bilateral lung contusion from blunt chest trauma in rats: cellular and cytokine responses. Shock 24: 132-138, 2005.

9. Kang SC, Matsutani T, Choudhry MA, Schwacha MG, Rue LW, Bland KI and Chaudry IH: Are the immune responses different in middle-aged and young mice following bone fracture, tissue trauma and hemorrhage? Cytokine 26: 223-230, 2004.

10. Wichmann MW, Ayala A and Chaudry IH: Severe depression of host immune functions following closed-bone fracture, soft-tissue trauma, and hemorrhagic shock. Crit Care Med 26: 1372-1378, 1998 .

11. Mimasaka S, Ohtsu Y, Tsunenari S and Funayama M: Postmortem cytokine levels and severity of traumatic injuries. Int J Legal Med 120: 265-270, 2006.

12. Taniguchi T, Koido Y, Aiboshi J, Yamashita T, Suzaki S and Kurokawa A: The ratio of interleukin- 6 to interleukin-10 correlates with severity in patients with chest and abdominal trauma. Am J Emerg Med 17: 548-551, 1999. 